NBER WORKING PAPER SERIES

\title{
ADOPTION CURVES AND SOCIAL INTERACTIONS
}

\author{
William A. Brock \\ Steven N. Durlauf \\ Working Paper 15065 \\ http://www.nber.org/papers/w15065

\section{NATIONAL BUREAU OF ECONOMIC RESEARCH 1050 Massachusetts Avenue Cambridge, MA 02138} \\ June 2009
}

This paper has been written in memory of Antoni Calvo-Armengol. The research has been supported by the National Science Foundation grant SES-0518274 and University of Wisconsin Vilas Trust and Graduate School. Thanks to Bryan Graham, Harald Uhlig and seminar participants at the memorial conference for Antoni Calvo-Armengol and the University of Chicago for very helpful comments on a previous draft. Nonarit Bisonyabut and Xiangrong Yu have provided superb research assistance. The views expressed herein are those of the author(s) and do not necessarily reflect the views of the National Bureau of Economic Research.

NBER working papers are circulated for discussion and comment purposes. They have not been peerreviewed or been subject to the review by the NBER Board of Directors that accompanies official NBER publications.

(C) 2009 by William A. Brock and Steven N. Durlauf. All rights reserved. Short sections of text, not to exceed two paragraphs, may be quoted without explicit permission provided that full credit, including (C) notice, is given to the source. 
Adoption Curves and Social Interactions

William A. Brock and Steven N. Durlauf

NBER Working Paper No. 15065

June 2009

JEL No. C1,D01,O33

\title{
$\underline{\text { ABSTRACT }}$
}

This paper considers the observational implications of social influences on adoption decisions for an environment of perfect foresight adopters. We argue that social influences can produce two observable effects: 1) discontinuities in unconditional adoption curves and 2) pattern reversals in conditional adoption curves, in which earlier adoption is found for one group of actors versus another when "fundamentals" suggest the reverse ordering should occur; in turn the presence of either of these features can, under weak assumptions, be interpreted as evidence of social influences. As such, these properties are robust implications of social effects.

\author{
William A. Brock \\ Department of Economics \\ University of Wisconsin \\ 1180 Observatory Drive \\ Madison, WI 537061393 \\ wbrock@ssc.wisc.edu \\ Steven N. Durlauf \\ Department of Economics \\ University of Wisconsin \\ 1180 Observatory Drive \\ Madison, WI 53706-1393 \\ and NBER \\ sdurlauf@ssc.wisc.edu
}




\section{Introduction}

There has been a resurgence of interest in the role of social interactions in determining the rate at which technologies are adopted (Bandiera and Rasul (2006), Conley and Udry (2005), Manski (2004,2006), Munshi (2004), Skinner and Staiger (2005), Young (2007)). Some of these recent efforts echo earlier debates that emerged when Griliches produced his seminal work on adoption in agriculture $(1957,1958)$. Griliches was criticized by Havens and Rogers (1961) among others for ignoring the social determinants of adoption decisions ${ }^{1}$. An important methodological implication of social interactions and adoption literature is that it shows that economic incentives and social influences may be synthesized so that any antithesis assumed between economic and social explanations is a false one; private incentives and social incentives are both compatible with the choice-based logic that Griliches developed.

This paper focuses on the properties of a particular rational expectations model of heterogeneous atomistic potential adopters. "Social interactions" in the context of this model constitute positive feedback external spillover effects from the fraction having already adopted in the payoff function to each agent who has adopted. Our objective is to identify properties of adoption curves that imply the presence of social interactions under relatively weak assumptions. Of course, any judgment on whether these assumptions are weak enough to be plausible will depend on context. In motivating our analysis in various places, we will return to the hybrid corn example; Skinner and Staiger (2005) and Sutch (2008) are recent studies that revisit Griliches' classic analysis in order to consider the effects on adoption. Of course the general literature on adoption of new technologies, network effects, learning effects, and the relationship of adoption to general social interactions is very large and had moved far beyond Griliches's early studies; see the survey by Hoppe (2002).

We characterize equilibria for rational adopters who maximize intertemporal profits. This characterization focuses on uncovering observational implications of social interactions on adoption curves that are robust to various types of observed and

\footnotetext{
${ }^{1}$ See David (2005) for a discussion of this debate.
} 
unobserved heterogeneity. In doing this, we are particularly concerned to identify observable implications that are robust to the presence of heterogeneity. To our knowledge the insertion of endogenous (in the sense of Manski (1993)) social interactions at the adoption level in the form of positive feedback of the fraction who have already adopted into the payoff function of each agent (after adoption) in the context of a rational expectations model of atomistic heterogeneous potential adopters and the study of the resulting patterns in adoption curves produced by this type of model is new. ${ }^{2} \mathrm{We}$ recognize that the adoption curve itself may of less interest than other features of patterns of adoption (e.g. delays in adopting superior technologies, patterns of strategic interaction between a small number of major players, etc.) may be considered more relevant, especially for policy making, we focus on properties of the adoption curve here because of their use in uncovering the presence of social influences which are of general interest and matter for policy questions.

Our analysis reveals two properties that might assist empirical researchers in interpreting some interesting patterns in the data. First, we demonstrate that social interactions can produce jumps in fraction adopting at some particular date. Second, we demonstrate that social interactions can produce pattern reversals in which agents whose private characteristics suggest they would adopt earlier than others in fact adopt later. Rather, it follows from the requirement that agents with greater ability to profit from a new technology adopt before those with relatively lesser ability. The reason that this requirement empirically distinguishes cases with and without social interactions is that under monotonic (in ability) adoption curves, lower ability agents face an environment in which a larger percentage of the population will have adopted than higher ability ones and hence may experience stronger social interaction effects because they adopt at a later date. In order to reconcile the difference in the strength of social interactions with the monotonicity requirement in adoption times and individual ability, discontinuities can occur. As for pattern reversals, the presence of discontinuities with in adoption curves with respect to unobservables can break the monotonicity with respect to observables.

${ }^{2}$ See Brock and Durlauf (2001a,b,2006,2007), Graham (2008), Lee (2007) and Manski (1993) for the econometric literature on social interactions. 
To be clear, neither of these properties is necessary for the presence of social interactions, and each is sufficient only in conjunction with additional assumptions on the adoption process. That said, the relatively weak nature of these assumptions (compared to others that appear in the literature) combined with the fact that these patterns only occur when social interactions are large (in a sense made precise below), provide strategies for empiricists to uncover social influences in adoption. ${ }^{3}$ While we certainly do not claim to have established that either discontinuities or pattern reversals constitute a sine qua non of observable implications of social interactions in adoption contexts, we do believe they represent useful directions for uncovering social influences on individual behavior. As such, they follow a research strategy in the social interactions literature developed in Brock and Durlauf (2007), Graham (2008) of trying to identify implications of social interactions are hold for an array of assumptions on unobserved heterogeneity and so may be regarded as robust implications.

Methodologically, two papers are closest to ours. Cabral (1990), in what appears to be a relatively neglected contribution, studies adoption curves when social interactions (in his language, network externalities) are present and shows how discontinuities in adoption curves may result. ${ }^{4}$ We differ from Cabral first in terms of our analysis of forward looking agents who face dynamic profit flows and second in our analysis of observable implications in the presence of various types of heterogeneity. ${ }^{5}$ Our analysis also shares much in common with Young (2007) who compares the properties of adoption models with and without different types of social interactions, and like us focuses on uncovering properties of adoption curves that are robust to heterogeneity. While our goals are similar, our microstructures and analyses are not. We analyze environments in which adopters are

${ }^{3}$ We thank an anonymous referee for this formulation.

${ }^{4}$ We became aware of Cabral's paper after writing the first draft of this one. We wish to emphasize Cabral's priority in uncovering the discontinuity property for adoption, despite differences in our microeconomic specifications.

${ }^{5}$ Other papers share important similarities with our analysis and Cabral's. De Palma (2007) studies a model of synchronization in the presence of social interactions. This analysis produces interdependences in hazard functions for individuals and in turn is related to Brock and Durlauf (2001b) and especially Sirakaya (2006) each of which directly embeds social interactions in a duration framework. Adsera and Ray (1998) consider a dynamic migration model with spillovers and show that lags in the effects of social interactions can eliminate multiple equilibria. 
forward looking whereas Young focuses on myopic adjustment rules that correspond to different types of social interactions. Heterogeneity in Young's analysis is associated with parameters of these adjustment processes whereas ours concerns observable and unobservable individual productivity. We find that social interactions produce different observable implications from those identified by Young. Unlike Young, we do not differentiate between types of social interactions.

We argue that one can derive some restrictions on adoption curves even when agents are forward looking and even quite general forms of heterogeneity are present. This will occur in two respects. First, we argue that social interactions can endogenously produce discontinuities in adoption curves. These discontinuities only arise exogenously when social interactions are absent. Second, we argue that social interactions can, in the presence of unobserved heterogeneity, reverse what would otherwise be a monotonic relationship between "fundamental" determinants of adoption and adoption times. Brock and Durlauf (2007) introduce the idea of pattern reversals in social interactions models. While we do not claim to have established that either discontinuities or pattern reversals constitute a sine qua non of observable implications of social interactions in adoption contexts, we do believe they represent essential directions for uncovering social influences on individual behavior.

The ability of discontinuities and pattern reversals to reveal social interaction effects in adoption curves does not rely on the existence of multiple equilibria, which is the source of the pattern reversal findings in Brock and Durlauf (2007). Rather, it follows from the requirement that agents with greater ability to profit from a new technology adopt before those with relatively lesser ability. The reason this requirement empirically distinguishes cases with and without social interactions is that lower ability agents face an environment in which a larger percentage of the population will have adopted than higher ability ones and hence may experience stronger social interaction effects. In order to reconcile the difference in the strength of social interactions with the monotonicity requirement in adoption times and individual ability, discontinuities and pattern reversals can occur. The uncovering of evidence of social interactions via multiple equilibria would require repeated observations of the same environment, so that differences in the 
equilibrium outcomes can be used to infer the presence of the multiplicity. No such mechanism is available here.

\section{Basic model}

We model potential adopters as rational farsighted individuals who choose adoption times. We claim no novelty for the microeconomic specification of this forward looking model with the exception of the introduction of social interactions. Variations on the model without social interactions appear in several papers in Punzo (2001); Brock (2001) also gives a review of some of this type of literature. In general, the social interactions literature has not dealt with dynamic environments with rational agents. Reinganum's pioneering work $(1981 \mathrm{a}, 1981 \mathrm{~b})$ is an important predecessor in terms providing examples of studies of adoption settings in which decisions are interdependent because of the competitive structure. Reinganum shows that this force alone (with no heterogeneity across individual actors) is enough to produce many Nash equilibria with heterogeneous adoption times. In contrast, our model focuses on social forces that lead to bunching in adoption times and explicitly considers the implications of heterogeneity. Bandiera and Rasul (2006) is a recent study that is complementary to our; they work with a social learning model in a linear environment.

Formally, we consider a population of individuals each of whom chooses an adoption time $t$ in order to maximize the present discounted value of current and future profits. We allow for heterogeneity across the individuals, by associating each individual with a scalar $x$ which may be interpreted as individual ability. Ability should be understood as indexing individual productivity; we take no stance on its determinants as it is treated as exogenous to the model. This heterogeneity is drawn from some continuously differentiable distribution function $F_{X}(x)$ with associated density $f_{X}(x)$. The support of $x$ is assumed to be an interval $I=[0, \hat{x}]$, where $\hat{x}$ may be infinite.

Individual actors choose adoption times $t$ in order to maximize 


$$
J(t, x)=\left(-\exp (-\rho t) C+\int_{t}^{\infty} \exp ((a-\rho) s) \pi\left(x, q^{e}(s)\right) d s\right)
$$

In this expression $\rho$ is the discount rate, $C$ is the cost of adoption, $a$ is the rate of technical progress, $\pi\left(x, q^{e}(s)\right)$ is expected profit flow at date $s$ (which depends upon the type $x$ ), where $q^{e}(s)$ is the expected fraction of adopters in the population who have adopted by date $s$. We assume that all agents have the same expectations. The dependence of profits on this fraction constitutes what we mean by social interactions. Relative to standard adoption models, the only innovation is the presence of $q^{e}(s)$ in the profit function. Our objective is to understand whether observed adoption behavior can reveal the role of social interactions in individual decisions.

We make the following assumptions on the elements of this decision problem.

Assumption A.1. $\rho-a>0$.

Assumption A.2. $q^{e}(t)$ is a piecewise differentiable monotone nondecreasing function with a finite number of points of discontinuity. For all $t$, right derivatives are well defined and left limits exist for $q^{e}(t)$.

Assumption A.3. $\pi(x, q)$ is strictly increasing in $x$, weakly increasing in $q$ and is twice continuously differentiable in $(x, q)$.

Assumption A.4. Each potential adopter of type $x$ does not take into account the impact of his choice of adoption time $t$ upon the adoption time choices of others.

Assumption A.1 is necessary to ensure that each individual's maximization problem is well defined; it simply ensures that the present discounted value of profits is bounded. Assumption A.2 imposes some regularity on the set of admissible beliefs which is useful in developing our results as it rules out potential pathologies. The monotonicity requirement 
is substantively quite restrictive. We make it in order to render the problem of characterization of the set of rational expectations equilibria tractable. ${ }^{6}$ We conjecture that monotone beliefs may be plausible in microfounded models where adopters keep learning about their new technology by newsletters, user groups, and other mechanisms of information transfer where the usefulness of the information transferred is increasing in the fraction that have already adopted the particular technology under scrutiny. Assumption A.3 restricts the payoff function and is therefore substantive in its impositions of monotonicity, but is of course far weaker than assuming a particular functional form. The main restriction is that the heterogeneity scalar $x$ has a monotonic effect on profits, which limits what sorts of interpretation may be placed on it. Assumption A.4 allows us to ignore strategic interactions, i.e. we treat each agent as atomistic. The assumption is sensible when an individual adopter is small relative to the overall group.

All propositions stated in the paper assume at least some of A.1-A.4; for ease of exposition we assume all of them for each of our lemmas and theorems. Further, we always assume that there is a finite date in the past when the new technology first appears and the first adoption occurs after that date; without loss of generality we date at which the innovation first appears at 0.7 Finally, we conceptualize each agent as choosing an adoption time from $(-\infty, T)$, this avoids problems of corner solutions in the optimization problem.

The first order necessary condition (FONC) and secondary order necessary condition (SONC) for the optimal adoption time $t^{*}$ by type $x$ are given by

${ }^{6}$ The assumption is consistent with the strategy of finding rational expectations equilibria that is standard in macroeconomics models in that we in essence conjecture that agents form beliefs that have certain properties and then show that these beliefs are justified in the equilibrium law of motion for the system.

${ }^{7}$ Sutch (2008) shows that this is precisely what happeed in the case of hybrid corn. Hybrid corn was invented by Donald F. Jones in 1917-1918, was developed and introduced on a trial basis by Henry Agard Wallace in 1924, was first sold commercially in 1925, competitors began sales in 1928, and widespread commercial adoption began in 1932. In 1933.1 percent of the nation's corn acreage was planted in hybrid corn and by 1960 hybrid corn was planted on 96.3 percent of the nation's corn acreage. 1917 corresponds to our $t=0$. 


$$
t^{*}(x)=\frac{1}{a}\left(\ln (\rho C)-\ln \left(\pi\left(x, q^{e}(t)\right)\right)\right)=0
$$

and

$$
-\rho^{2} C \leq \exp (a t)\left((a-\rho) \pi\left(x, q^{e}(t)\right)+\frac{\partial \pi\left(x, q^{e}(t)\right)}{\partial q^{e}(t)} \frac{d q^{e}(t)}{d t}\right)
$$

respectively for dates $t$, if $q^{e}(t)$ is differentiable at $t$. However, as will become clear, we do not wish to assume differentiability at all dates. If $q^{e}(t)$ is not differentiable at date $t$, observe that by A.3 $q^{e}\left(t^{-}\right)<q^{e}\left(t^{+}\right)$where " -" denotes the operation of taking the left limit and "+" denotes the operation of taking the right limit. A local maximum is characterized (in our case) by the left limit being greater than the right derivative with a zero between these two values. Letting $J(t, x)$ denote the objective function in (1) evaluated at the particular point $(t, x)$. Then the first and second order necessary conditions for a local maximum at $t$ may be expressed as

$$
\begin{aligned}
& J^{\prime}\left(t^{-}, x, q^{e}\left(t^{-}\right)\right)=e^{-\rho t}\left(\rho C-e^{a t} \pi\left(x, q^{e}\left(t^{-}\right)\right)\right) \geq \\
& \quad 0 \geq \\
& J^{\prime}\left(t^{+}, x, q^{e}\left(t^{+}\right)\right)=e^{-\rho t}\left(\rho C-e^{a t} \pi\left(x, q^{e}\left(t^{+}\right)\right)\right)
\end{aligned}
$$

since this inequality can be decomposed in terms of the usual FONC and SONC, $J^{\prime}(t, x)=0, J^{\prime \prime}(t, x) \leq 0$ when $q^{e}(t)$ is differentiable at $t .{ }^{8}$

\footnotetext{
${ }^{8}$ Throughout, we write $t^{-}=t=t^{+}$for the parts of functions that are continuous in $t$.
} 
In the case where $q^{e}(t)$ is differentiable at date $t$, the FONC is quite intuitive as it amounts to equating the marginal benefit to adoption at a given time with the associated marginal cost. To understand the SONC, rewrite (2) as

$$
\rho C=\exp (a t) \pi\left(x, q^{e}(t)\right)
$$

Substituting into (3), the second order condition holds if and only if,

$$
0 \leq a \pi\left(x, q^{e}(t)\right)+\frac{\partial \pi\left(x, q^{e}(t)\right)}{\partial q^{e}(t)} \frac{d q^{e}(t)}{d t}
$$

Equation (6) is satisfied provided that $\pi\left(x, q^{e}(t)\right) \geq 0 \frac{\partial \pi\left(x, q^{e}(t)\right)}{\partial q^{e}(t)} \geq 0$ and $\frac{d q^{e}(t)}{d t} \geq 0$. The first inequality is immediate from the FONC, since the cost of adoption is positive. The second inequality holds by Assumption A.3 above. The third inequality cannot be assessed without specification of the expectations formation process. In our subsequent analysis $\frac{d q^{e}(t)}{d t} \geq 0$ will be shown to hold in equilibrium under rational expectations. In the case where $q^{e}(t)$ is not differentiable at $t$ we will show that $q^{e}\left(t^{-}\right) \leq q^{e}\left(t^{+}\right)$holds in equilibrium. Hence it will be the case that the SONC holds for interior critical points $t$ for (2). If $\rho C<\pi\left(x, q^{e}(0)\right)$, it is locally optimal to set $t=0$ for type $x$.

The following lemma provides a sufficient condition for uniqueness of an individual adoption time.

\section{Lemma 1. Uniqueness of optimal adoption time}

Given, A.1-A.4, the optimal time to adopt for each agent type exists and is unique. 
For comparative purposes, we note the case where there are no social interactions, i.e. $\pi\left(x, q^{e}(t)\right)=\pi(x)$. In this case the optimal adoption times follow

$$
\begin{gathered}
t^{*}(x)=\frac{1}{a}(\ln (\rho C)-\ln \pi(x)) \text { if } x<\bar{x}, \\
t^{*}(x)=0 \text { if } x \geq \bar{x}
\end{gathered}
$$

where $\bar{x}$ is defined by $t^{*}(\bar{x})=0$ (if such an $\bar{x}$ exists). Observe that $t^{*}(x)$ is decreasing in $x$ by Assumption A.3; this simply means that higher productivity types adopt earlier as the profit incentives are higher.

\section{Adoption curves and rational expectations equilibria}

We now consider equilibria under social interactions by imposing a rationality requirement on beliefs about adoption levels. For any expectations process, one can construct the actual adoption curve $q(t)$ that describes the percentage of the population which has adopted by date $t$. We first note a lemma.

\section{Lemma 2. Monotonicity of adoption with respect to ability}

Given A.1-A.4, suppose $t<t_{1}$ and $x<x_{1}$. If $x_{1}$ adopts at time $t_{1}$, then for all $x<x_{1}$, the adoption time $t$ of type $x$ satisfies $t \geq t_{1}$.

Monotonicity means that the adoption rate may be calculated using the formula

$$
q(t)=\int_{a(t)}^{\infty} f_{X}(z) d z=1-F_{X}(a(t))
$$

where the lower integral limit $a(t)$ is implicitly defined by 


$$
t^{*}(a(t))=t
$$

Eq. (8) means that there is a tight link between the adoption curve and the distribution function of the type-specific heterogeneity. To understand eq. (9), recall that we have shown that for each $x$ there exists a unique adoption time $t^{*}(x)$, a decreasing function which may be discontinuous, i.e. $t^{*}\left(x^{-}\right)>t^{*}\left(x^{+}\right)$may occur. At continuity points of $t^{*}(x)$, the associated inverse function is well defined and one can solve for the value $x=a(t)$ that satisfies (9) at date $t$. At discontinuity points of $t^{*}(x)$ we use the right limit $x^{+}$as $x$ decreases towards the point of discontinuity. This choice rule is consistent with Assumption A.2; this ensures that $q^{e}(t)$ is increasing in $t$.

Since the profit functions are assumed to depend on the expected adoption curves of each agent, rational expectations equilibria thus are straightforward to define as they require that the beliefs about adoption rate coincide with the actual adoption rates along an equilibrium path.

\section{Definition: rational expectations equilibrium (REE).}

A rational expectations equilibrium is a pair of functions $t^{*}(x)$ and $q^{*}(t)$ such that

i. individual adoption times are optimal,

and

ii. the aggregate adoption curve is consistent with these individually optimal choices.

The requirements of a rational expectations equilibrium, implicitly characterizes the optimal adoption times. At points of differentiability, the optimal times must fulfill 


$$
t^{*}(x)=\frac{1}{a}\left(\ln (\rho C)-\ln \left(\pi\left(x, q^{*}(t)\right)\right)\right) \text { if } t^{*}(x)>0
$$

and the equilibrium fraction who have adopted by date $t, q(t)$, must fulfill

$$
q^{*}(t)=\operatorname{Pr}\left(x \mid \rho C \exp (-a t) \leq \pi\left(x, q^{*}(t)\right)\right)=1-F_{X}\left(x^{*}(t)\right)
$$

where $x^{*}(t)$ is implicitly defined by the first equality in (11). Substituting (11) into (10)

$$
t^{*}(x)=\frac{1}{a}\left(\ln (\rho C)-\ln \pi\left(x, 1-F_{X}\left(x^{*}(t)\right)\right)\right)
$$

In contrast to this function, one can consider a best response function for each type in which the population fraction adopting corresponds to the distribution of types:

$$
S(x)=\frac{1}{a}\left(\ln (\rho C)-\ln \pi\left(x, 1-F_{X}(x)\right)\right) .
$$

This $S(x)$ function differs from $t^{*}(x)$ as it is the best response function for an individual without self-consistency imposed between the adoption time and adoption curves as an equilibrium condition.

\section{Restrictions on the shape of the adoption curve}

In this section, we consider how social interactions manifest themselves in observed adoptions decisions. We follow the tradition started with Griliches (1957) in placing primary emphasis on the shape of the equilibrium adoption curve $q^{*}(t)$. We consider how social interactions, in the sense that the profit function $\pi(x, q)$ increases in both $x$ 
and $q$ restricts this curve. Our goal is to understand how restrictions emerge in light of heterogeneity as characterized by $F_{X}(x)$ and the unobservability of $\pi(x, q)$. In other words, we are interested in uncovering empirical implications of social interactions that are robust to unobserved heterogeneity in terms of individual types and the associated profit functions that characterize agents.

In order to develop restrictions on the shape of the adoption curve which are generateed by social interactions, we focus on eq. (13). When social interactions are absent, $\pi(x, q)=\pi(x)$ and so is monotonically increasing in $x$ by Assumption A.3; this implies that $S(x)$ is monotonically decreasing as well. On the other hand, when social interactions are present the $\pi\left(x, 1-F_{X}(x)\right)$ in (13) is not necessarily increasing in $x$ despite Assumption A.3 because $1-F_{X}(x)$ is decreasing in $x$; by (13) nonmonotonicity may be transferred to $S(x)$. Intuitively, this means that, as a best response, higher ability agents may not find it as profitable to adopt as lower ability agents because of the absence of others who adopt at the same or earlier times. The breakdown, in the presence of social interactions, of the monotonicity of the profit function with respect to $x$ and associated nonmonotonicity of $S(x)$ has important implications for the behavior for the observable, $q^{*}(t)$

We start by concentrating on a form of $S(x)$ such that $S(0)=\infty$, when $x$ is small, with a unique minimum $\underline{x}$. Let $x_{1}(t)$ denote the smallest and $x_{2}(t)$ the largest solutions to $t=S(x)$. We wish to demonstrate that $x_{1}(t)$ can be part of a rational expectations equilibrium, but that $x_{2}(t)$ cannot. Suppose that $x_{2}(t)$ is part of an REE. At $x_{2}(t)$, higher $t^{*}$ values are associated with larger $x$ values. This solution is not one that will be produced by agents choosing optimally conditional on their commonly shared belief that $x_{2}(t)$ is an equilibrium; this contradiction follows from Assumption A.3 that partial derivative of the profit function with respect to $x$ is positive. Hence only $x_{1}(t)$ has 
the potential to be part of an REE solution. This type of argument generalizes to produce Lemma 3; the proof is trivial given our previous results and is therefore omitted.

\section{Lemma 3. Monotonicity along equilibrium adoption paths}

Given A.1-A.4, along an equilibrium adoption path $q^{*}(t)=1-F_{X}\left(x^{*}(t)\right)$,

A. the process for optimal adoption times $t^{*}(x)$ must obey $\frac{d x}{d t}<0$ for points of differentiability.

B. At jumps, larger $x$ types adopt earlier than smaller $x$ types.

How does the possibility of nonmonotonicity of $S(x)$ interact with the requirement that $\frac{d x}{d t}<0$ in equilibrium? The reconciliation of these requirements places restrictions on $q^{*}(t)$. Intuitively, in order to ensure that $\frac{d x}{d t}<0$, at differentiable times it is necessary that for higher $x$ 's, a sufficient number of adopters are present to ensure that adoption is monotonic in type. In order for this to always hold, it may be necessary for the $q^{*}(t)$ function to exhibit discontinuities. This can produce discontinuities of the following form: as $t$ increases from slightly below $t^{*}$ to slightly above $t^{*}$, a mass point of size $F_{X}\left(x^{*}\right)-F_{X}\left(x^{\prime}\right)>0$ adopts where $x^{\prime}<x^{*}$ is defined by

$$
S\left(x^{\prime}\right)=S\left(x^{*}\right)
$$

This jump in the number of adopters means that the $t^{*}(x)$ is never increasing in $x$. The adoption of a mass point at date $t^{*}$ causes a jump of size 


$$
q\left(t^{+}\right)-q\left(t^{-}\right)=F_{X}\left(x^{*}\right)-F_{X}\left(x^{\prime}\right)>0,
$$

to occur at time $t=t^{*}$. Hence an observable implication of social interactions, when the interactions are strong enough to induce a nonmonotonicity in the function $S(x)$ in $x$, is that there may exist at least one jump in $q^{*}(t)$.

As our objective is to explicitly link discontinuities in the REE function $q^{*}(t)$ to the identification of social interactions, we formulate a theorem for the particular case of $S(x)$ in which the function has a single local minimum $x_{\min }$ and a single finite local maximum $x_{\max }$; Figure 1 illustrates the qualitative shape of $S(x)$ that is assumed in the theorem The structure of the theorem makes it evident that other formulations are possible. While we conjecture that a more general theorem may be produced which directly maps the nonmonotonicity of $S(x)$ to discontinuities in $q^{*}(t)$, we have yet to show this; the difficulty is that in dynamic models, one needs to evaluate the best reply function against the full dynamic path of the associated adoption curve. A parametric example of the theorem is provided in the Appendix.

\section{Theorem 1. Discontinuities in the adoption curve as evidence of social interactions}

Given A.1-A.4, suppose that

i. $S(x)$ is continuously differentiable in $x, S(0)=\infty$,

ii. $S(x)$ is initially strictly decreasing in $x$, until it takes a positive local minimum at $x_{\min }$,

iii. $S(x)$ increases in the open interval $\left(x_{\min }, x_{\max }\right)$, 
iv. $S(x)$ takes a unique local maximum at $x_{\max }>x_{\min }$, and then strictly decreases in $x$ for all $x>x_{\max }$ until some point $\tilde{x}>x_{\max }$ is reached

v. For all $x>\tilde{x}, S(x)<0$.

Define $x_{*}<x_{\min }<x_{\max }$ via $t^{*}=S\left(x_{\max }\right)=S\left(x_{*}\right)$. Then,

A. There exists an REE, $q^{*}(t)$ defined by, $q^{*}(t)=1-F_{X}\left(x^{*}(t)\right)$, for which $x^{*}(t)$ satisfies the equation,

$$
t=S\left(x^{*}(t)\right)
$$

where $x^{*}(t)$ is defined to be the largest solution, $x_{3}(t)$, to the equation $t=S(x)$, for $t \leq t^{*}$ and $x^{*}(t)$ is defined to be the unique solution, $x_{1}(t)$, to the equation $t=S(x)$, for $t>t^{*}$ where $t^{*}=S\left(x_{\max }\right)$.

B. There exists one jump point of positive size in the equilibrium adoption curve $q^{*}(t)$ which occurs at $t^{*}=S\left(x_{\max }\right)$,

C. There is a mass point, $1-F_{X}(\tilde{x})$, at $t=0$.

This discontinuity property represents a variant of a partial identification argument in econometrics, cf. Manski (2008). By this, we mean that the presence of social interactions can, for certain magnitudes of the interactions, place restrictions on observable data, but that these restrictions neither identify the magnitude of the interactions (in this case the effects of $q$ on $\pi(x, q)$ nor are the restrictions necessary for the interactions to be present. The presence of a discontinuity means that in comparing the adoption model 
with and without social interactions, i.e. comparing the implications of the dependence of the profit function on $q$, a discontinuity may be interpreted as evidence of social interactions. However, the absence of a discontinuity does not imply that social interactions are absent. The discontinuity property requires that the strength of the influence of social interactions is large. Given the absence of any restrictions on $\pi(x, q)$ beyond Assumption A.2, one cannot be more precise about what is meant by larger; if a functional form were chosen in which a parameter measured the strength of social interactions, then one could interpret the presence of discontinuities as a statement about the parameter's magnitude. "Large" is model-specific.

Of course, discontinuities in $F_{X}(x)$ can produce discontinuities in $q^{*}(t)$ even when social interactions are absent. If individual adoption times and associated values of $x$ are observable (so that $F_{X}(x)$ is observable), this alternative explanation can be assessed as any discontinuity at $t_{d}$ would have to align with a discontinuity at $x_{d}$ so that $t^{*}\left(x_{d}\right)=t_{d}$. An example in the hybrid corn context is the date 1936 when the news that hybrid corn produced much more then open pollinated varieties in that drought year spread like wildfire through the farming community and caused the demand for hybrid seed to explode (Sutch (2008, page 18)). This would look like a jump in the data but would not be due to social interactions in the sense that the jump is not caused by $\frac{\partial \pi}{\partial q}>0$. Of course if one conditions on relative yield information in 1936, separation of causes of jumps in adoption rates due to $\frac{\partial \pi}{\partial q}>0$ in contrast to an exogenous driver of jumps (such as information on the relative productivity of hybrid corn in drought conditions) should be possible. ${ }^{9}$

${ }^{9}$ One might argue that in the hybrid corn historical record our model is most relevant to the period after the 1936 jump. Following Sutch (2008), this is so because of the combination of the key initial role of Henry Agard Wallace in promoting the use of hybrid corn and the fact that did not dramatically expand until prompted by the news of the very high relative yields in the drought year, 1936. Sutch discusses the continued improvement of varieties of hybrid corn, the role of demonstration plots, the sharing of information 
Once one introduces individual heterogeneity, it is possible to develop empirical implications of social interactions which do not rely on the presence of jumps. For example, if one observes the adoption under two different distributions of observed heterogeneity, one of which stochastically dominates the other, the absence of social interactions places strong restrictions on the associated adoption curves.

\section{Theorem 2. Stochastic dominance and the absence of social interactions}

Suppose that the distribution function $F_{X, 1}(\cdot)$ is stochastically dominated by the distribution function $F_{X, 2}(\cdot)$. If there are no social interactions, i.e. $\pi(x, q)=\pi(x)$, then it must be the case that $q_{1}(t) \leq q_{2}(t)$ for all $t$.

The application of this Theorem would require that a research is able to identify distinct cases of adoption under different $F_{X, i}(\cdot)$ 's.

\section{Unobservable heterogeneity}

In this section we introduce unobservable heterogeneity and consider how empirical implications are affected. Interestingly, unobservable heterogeneity can produce a different route to uncovering social interactions that is not present when the heterogeneity is absent.

We focus on the case of a scalar observable $x_{1}$ and a scalar unobservable $x_{2}$. Before we begin a detailed analysis for the case of one observable and one unobservable we wish to indicate that the approach extends easily to the case of multiple observables and

about tailoring varieties to specific land types and the role of agricultural extension offices in the diffusion process of hybrid corn. 
multiple unobservables. This will follow from an assumption of monotonicity of $\pi$ in both observables and unobservables.

It is useful to recall the following elementary result from functional separability theory in general utility theory (Varian (1992, p. 150)): For vector $x$ and scalar $q$, if 1 ) $\pi(x, q)$ is strictly increasing in both arguments and 2) $\pi(x, q) \geq \pi\left(x^{\prime}, q\right)$ if and only if $\pi\left(x, q^{\prime}\right) \geq \pi\left(x^{\prime}, q^{\prime}\right)$ for all $x, x^{\prime}, q$ and $q^{\prime}$, then there exists a "subprofit" function $u(x)$ (mapping $x$ to a scalar) and an aggregator function $V(u, q)$ such that

$$
\pi(x, q)=V(u(x), q)
$$

If $V(u, q)$ is strictly increasing in $(u, q)$ one may simply repeat our earlier analysis by replacing $x$ in the above analysis with $u$. Hence in an REE, it must be the case that, $q=1-F_{U}(u)$. In an REE, when $V(u, q)$ is independent of $q$, individuals with higher levels of $u$ adopt first. When $V(u, q)$ varies in $q$, the best response adoption times for individuals with level $u$ is given by

$$
S(u)=\frac{1}{a}\left(\ln \rho C-\ln V\left(u, 1-F_{U}(u)\right)\right)
$$

In parallel to our earlier discussion, the unconditional equilibrium adoption curve is given by

$$
q^{*}(t)=1-F_{U}(t)
$$

where

$$
\begin{gathered}
t^{*}(u)=\frac{1}{a}\left(\ln (\rho C)-\ln \pi\left(u^{*}(t), 1-F_{U}\left(u^{*}(t)\right)\right)\right) \text { if } t^{*}(u)>0 \\
t^{*}(u)=0 \text { if } \ln \left(\pi\left(u, q^{*}(0)\right)\right) \geq \ln (\rho C) .
\end{gathered}
$$


Functional separability provides a general strategy for introducing unobservables. Suppose that productivity is a vector rather than a scalar attribute. Partition the vector as $x=\left(x_{1}, x_{-1}\right)$ where $x_{1}$ is observable and $x_{-1}$ is not. We may now repeat our earlier analysis to conditional adoption curves $q^{*}\left(t \mid x_{1}\right)$. These curves must obey

$$
q^{*}\left(t \mid x_{1}\right)=1-F_{U \mid X_{1}}\left(u\left(t \mid x_{1}\right)\right)
$$

We now focus on the case of a scalar unobservable, i.e. $x=\left(x_{1}, x_{2}\right)$, where each component is one dimensional. Notice that if one had infinite data on each "cell" $x_{1}$, i.e. one observes the conditional adoption curve $q\left(t \mid x_{1}\right)$ for every $x_{1}$, then this would be enough to "reveal" the one dimensional unobservable $x_{2}$ except at those dates when nonmonotonicity of the conditional best response time $S\left(x_{2} \mid x_{1}\right)$ occurs. To put it another way if the function $S\left(x_{2} \mid x_{1}\right)$ is one-to-one for all $x_{1}$ then knowledge of the conditional adoption curves at $t$ and the observable covariate $x_{1}$ reveals the unobservable $x_{2}$. On the other hand when the function $S\left(x_{2} \mid x_{1}\right)$ is not monotone in $x_{2}$ for some $x_{1}$ then our results derived above on the characterization of REE's apply, i.e. there will typically be a discontinuous burst of adoption as time $t$ passes through a critical value where the function is not monotone for this particular value of $x_{1}$.

Without any restrictions on the unobservables, one cannot identify any observable implications for social interaction effects on adoption curves. Any adoption pattern that is generated with social interactions can be replicated with them by suitable choice of unobservables. We proceed by using a "weak" assumption on the unobservables:

A.5. $F_{X_{2} \mid X_{1}}\left(x_{2}\right)$ is stochastically increasing in $x_{1}$ in the sense, $x_{1}<x_{1}^{\prime}$, implies that 


$$
F_{X_{2} \mid X_{1}}\left(x_{2} \mid x_{1}\right) \geq F_{X_{2} \mid X_{1}}\left(x_{2} \mid x_{1}^{\prime}\right) .
$$

Stochastic dominance is an example of a shape restriction. Shape restrictions are not only weaker than functional form restrictions, they are often interpretable in economic terms in ways that functional forms are not. For example, if one thinks of individual farmers as characterized by an unobservable ability level and an observable education level, then stochastic dominance says nothing more than higher education levels imply that the density of abilities is shifted to the right. Brock and Durlauf (2007) show how stochastic dominance can facilitate identification of social interactions, using partial identification arguments. This work, in turn, draws from Manski (1997) and Manski and Pepper (2000) who emphasize the constructive role of assumptions such as shape restrictions.

\section{Theorem 3. Monotonicity of adoption curves with respect to observables.}

Given A.1-A.5, suppose that both observables and unobservables, $x_{1}, x_{2}$ are one dimensional.

A. If there are no social interactions, i.e. $\frac{\partial \pi\left(x_{1}, x_{2}, q\right)}{\partial q}=0$, then if (22) holds

$$
x_{1}<x_{1}^{\prime} \text { implies } q\left(t \mid x_{1}\right)<q\left(t \mid x_{1}^{\prime}\right) \text {, for all } t \geq 0 \text {. }
$$

B. If

$$
x_{1}<x_{1}^{\prime} \text { but } q\left(t \mid x_{1}\right)>q\left(t \mid x_{1}^{\prime}\right) \text {, }
$$

then it must be the case that $\frac{\partial \pi\left(x_{1}, x_{2}, q\right)}{\partial q} \neq 0$. 
The theorem is intuitive. One expects agents with higher values of observable characteristics $x_{1}$ to have better values of unobservable characteristics on average and, hence, to have higher profits on average which cause more of them to adopt at any given observed date. Unless there is some kind of externality in the profit function, i.e. dependence on $q$, pattern reversals require that unobserved and observed heterogeneity move in opposite directions, which is ruled out by the stochastic dominance assumption.

Other routes exist to using pattern reversals as the basis for uncovering social interactions. Theorem 4 is one example; the proof is analogous to the proof of Theorem 3 and is therefore omitted.

\section{Theorem 4. Pattern reversals and order reversals}

Suppose there exists a time $t_{0}$ such that two different observable types adopt at this time. Suppose that at this $t_{0}$,

$$
q\left(t_{0} \mid x_{1}\right)>q\left(t_{0} \mid x_{1}^{\prime}\right)
$$

i.e. more of the less educated types adopted by $t_{0}$ than the more educated types. Then either $F_{X_{2} \mid X_{1}}\left(x_{2} \mid x_{1}\right)<F_{X_{2} \mid X_{1}}\left(x_{2} \mid x_{1}^{\prime}\right)$ or $\frac{\partial \pi\left(x_{1}, x_{2}, q\right)}{\partial q} \neq 0$.

As a special case of the Theorem, if $x_{1}$ and $x_{2}$ are independent, then a pattern reversal is interpretable as evidence of social interactions.

Theorem 4 assumes a one-dimensional unobservable, but is more general than it appears on the surface. For an $n$-dimension unobservable, denote the individual profit function as $\pi\left(x_{1}, x_{-1}, q\right)$ where $x_{-1}=\left(x_{2}, \ldots, x_{n}\right)$ and assume that the profit function is increasing in all arguments. To adapt the argument to the higher dimension vector of unobservables, assume there exist monotone increasing scalar valued functions $V$ and $u$ 
such that $\pi\left(x_{1}, x_{-1}, q\right)=V\left(x_{1}, u\left(x_{-1}\right), q\right)$. One can then replace the scalar $x_{-1}$ in the argument leading to Theorem 4 with the scalar $u\left(x_{-1}\right)$ and obtain a generalization of Theorem 4 to an arbitrary number of unobservables; the last part of Theorem 4 generalizes to the requirement that either $F_{U \mid X_{1}}\left(u \mid x_{1}\right)<F_{U \mid X_{1}}\left(u \mid x_{1}^{\prime}\right)$ or $\frac{\partial V\left(x_{1}, u\left(x_{2}\right), q\right)}{\partial q} \neq 0$. Of course, the functional separability of payoffs with respect to a vector of unobservables may be more difficult to justify based on heuristic economic reasoning.

\section{Comparisons to other approaches to uncovering social interactions in adoption}

In this section, we consider some alternate approaches which have been proposed for uncovering social interactions

\section{A. logistic functions and adoption curves}

It is often claimed that logistic adoption curves are evidence of social interactions; Schelling (1997) provides an overview of interpretations of logistic curves as evidence of social effects. This type of argument typically derives from a view of social interactions as a type of infection phenomena in which the fraction of those who have not adopted, $1-q(t)$, changes via

$$
\frac{d q(t)}{d t}=R q(t)(1-q(t))
$$

See Daley and Gani (2001) for discussion of this equation in epidemiology. In our model, the derivative of the adoption curve is 


$$
\frac{d q(t)}{d t}=-f_{X}(x)\left(\frac{d S(x)}{d x}\right)^{-1}=R\left(1-F_{X}(x)\right)\left(F_{X}(x)\right)
$$

and can be constructed by solving the ordinary differential equation,

$$
S^{\prime}(x)=\frac{f_{X}(x)}{R\left(1-F_{X}(x)\right) F_{X}(x)}
$$

But it follows from the definition of $S(x)$ that

$$
S^{\prime}(x)=-\left(\frac{1}{a}\right) \frac{\pi^{\prime}(x)}{\pi(x)}
$$

Hence, given $f_{X}(x)$ (and the associated distribution function $F_{X}(x)$, in order to have a logistic dynamic (26) generated by our model all one must do is to construct a $\pi(x)$ function, to satisfy the equation,

$$
\ln (\pi(x))-\ln \left(\pi\left(x_{0}\right)\right)=\frac{a}{R} \int_{x_{0}}^{x} \frac{f_{X}(x)}{\left(\left(1-F_{X}(x)\right) F_{X}(x)\right)} d x
$$

for some lower bound $x_{0}$. This indicates how logistic dynamics can result from the shape of the profit function $\pi(x)$ with no implication about social interactions in the adoption process. Since the profit function is not observable, one cannot use the shape of the adoption curve to infer anything about social interactions. Put differently, $\pi(x)$ represents a latent variable that varies across types and is itself a form of unobserved heterogeneity; eq. (31) shows that the mapping of a logistic curve to social interactions is not robust when this type of heterogeneity is present. 
One can develop a parallel analysis to demonstrate that, in the context of our model, logistic adoption curves may be generated in absence of social interactions ${ }^{10}$. Suppose that the profit function does not embody social interactions and has the form

$$
\pi(x, q)=A x^{\alpha}, 0<\alpha<1
$$

Mimicing our earlier arguments, profit maximization implies that equilibrium adoption times are implicitly defined by

$$
x(t)=(\rho C / A)^{1 / \alpha} e^{-(a / \alpha) t}
$$

Previous arguments establish that $\frac{d q}{d t}=-f(x) \frac{d x}{d t}$ therefore (32) implies $\frac{d x}{d t}=-\frac{a x}{\alpha}$. Further, (26) indicates the necessary condition for a logistic adoption curve. Combining these yields the following differential equation for $\mathrm{F}(\mathrm{x})$

$$
\frac{a}{\alpha} \frac{d F(x)}{F(x)(1-F(x))}=r \frac{d x}{x}
$$

Integrating both sides of (33) (using partial fractions for the LHS) one obtains the solution

$$
F(x)=\frac{c_{0} x^{r \alpha / a}}{1+c_{0} x^{r \alpha / a}}, c_{0}>0
$$

and

${ }^{10}$ See Manuelli and Seshadri (2008) for a distinct framework in which $S$-shaped adoption curves are produced without social interactions, which captures the qualitative features of the logistic function, the importance of which is discussed below. 


$$
q(t)=\frac{d_{0}}{1+d_{0} e^{-r t}}
$$

where the constant $d_{0}:=c_{0}(\rho C / A)^{r / a}$. This is a logistic function. The logistic shape for this case derives from the density of types, $F(x)$, not from the presence of interactions. One can therefore object that this is a knife edge case. However, as noted by Feller (1940) and further argued in Brock (1999) and Dinardo and Winfree (2007), it is very difficult to distinguish logistic curves from $S$-shaped alternatives, hence the qualitative properties of the logistic are derivable from a range of densities.

\section{B. relative acceleration rates}

Young (2007) is a recent effort to use adoption curve shapes to uncover social interactions. His analysis is based on the function

$$
g(r)=\frac{d^{2} q(t)}{d t^{2}} / \frac{d q(t)}{d t} \text { evaluated at } t_{r} \text { where } q\left(t_{r}\right)=r \text {. }
$$

which he calls the "relative acceleration rate when $r$ is the proportion of the population that has already adopted." Young argues that in one type of social interactions model, a social learning process, the relative acceleration rate "is often nonlinear: rising in the early phases of adoption and declining in the later phases. In another type of social interactions model, a contagion process, by contrast, he argues that the relative acceleration rate is typically level or declining from beginning to end.” (Young (2007, p. 4)).

To understand the behavior of the relative acceleration rate in our model, algebraic manipulation reveals that, even when social interactions are absent, the relative acceleration rate for our model is 


$$
g(r)=\frac{d f_{X}\left(x_{r}^{*}(t)\right)}{d x}\left(f_{X}\left(x_{r}^{*}(t)\right) \frac{d t^{*}\left(x_{r}^{*}(t)\right)}{d x}\right)^{-1}-\left(\frac{d^{2} t^{*}\left(x_{r}^{*}(t)\right)}{d x^{2}}\right)\left(\frac{d t^{*}\left(x_{r}^{*}(t)\right)}{d x}\right)^{-2}
$$

where the quantities are evaluated at $x^{*}\left(t_{r}\right)$. Additional algebraic manipulation produces

$$
\begin{gathered}
g(r)= \\
a \pi\left(x_{r}^{*}(t)\right)\left(\frac{d \pi\left(x_{r}^{*}(t)\right)}{d x}\right)^{-1}\left(\frac{d f_{X}\left(x^{*}(t)\right)}{d x}\right) \times \\
\left(\left(\frac{d f_{X}\left(x^{*}(t)\right)}{d x}\right) f_{X}\left(x^{*}(t)\right)+\pi\left(x_{r}^{*}(t)\right)\left(\frac{d \pi\left(x_{r}^{*}(t)\right)}{d x}\right)^{-1}\left(\frac{d^{2} \pi\left(x_{r}^{*}(t)\right)}{d x_{2}}-\left(\frac{d \pi\left(x_{r}^{*}(t)\right)}{d x}\right)\right) .\right.
\end{gathered}
$$

For our purposes, what is important about this expression is that one can manufacture a wide variety of shapes of $g(r)$ by varying the values of $\frac{d f_{X}(x)}{d x}$ and $\frac{d \pi^{2}(x)}{d x^{2}}$. Economic theory of course does not restrict these functions. Hence, the relative acceleration rate is not restricted by our forward looking model. By implication, one cannot distinguish types of social interactions, i.e. contagion versus social learning. Again, $\frac{d f_{X}(x)}{d x}$ and $\frac{d \pi^{2}(x)}{d x^{2}}$ are forms of unobserved heterogeneity that break any logical link between the shape of the adoption curve and social interactions.

\section{Conclusions}

In this paper we have analyzed a model of adoption decisions in which social interactions are present. Our analysis indicates that even in the presence of observable and unobservable heterogeneity, it is possible to uncover properties of adoption curves that observationally differentiate environments in which social interactions matter from those that do not. While we have not translated these observational differences into econometric 
analogs, their presence provides a basis for constructing formal econometric tests. Work in the time series literature and finance literatures on the identification of jumps in stochastic processes, e.g. Ait-Sahalia (2004), Ait-Sahalia and Jacod (2008) and Barndoff-Neilson and Shephard (2006), suggests that such testing is possible. 
Figure 1

Shape of $S(x)$ in Theorem 1

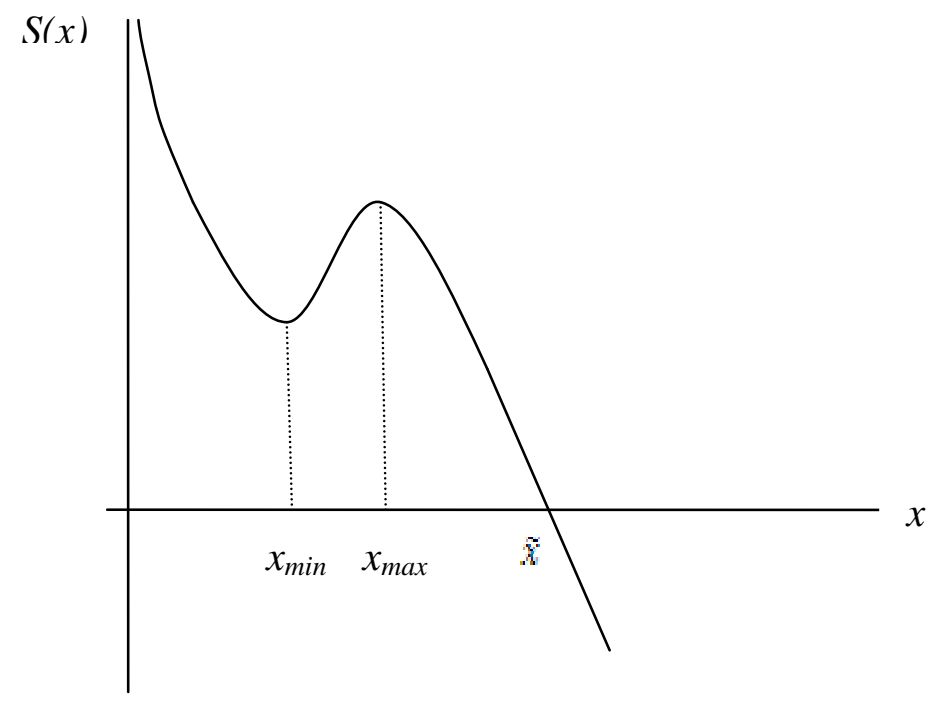




\section{Appendix 1: Proofs of Theorems}

\section{Proof of Lemma 1.}

Assumption A.1 implies that $\pi\left(x, q^{e}(t)\right) \leq \pi(x, 1) \forall t$. Suppose by way of contradiction that there exist two optimal times $t_{1}<t_{2}$ such that $J\left(t_{1}, x, q^{e}(t)\right)=J\left(t_{2}, x, q^{e}(t)\right)$. There are four cases to consider (i) $q^{e}(t)$ is differentiable at both $t^{\text {s }}$, (ii) $q^{e}(t)$ is differentiable at $t_{1}$ but not $t_{2}$, (iii) $q^{e}(t)$ is differentiable at $t_{2}$ but not $t_{1}$, (iv) $q^{e}(t)$ is not differentiable at either $t$. For each of the cases, $J^{\prime}\left(t_{i}^{-}, x, q^{e}\left(t_{i}^{-}\right)\right) \geq 0 \geq J^{\prime}\left(t_{I}^{+}, x, q^{e}\left(t_{i}^{+}\right)\right)$by eq. (4). Using the representation in (4) and cancelling off $e^{-\rho t}$, it must be the case that

$$
\rho C-e^{a t_{i}} \pi\left(x, q^{e}\left(t_{i}^{-}\right)\right) \geq 0 \geq \rho C-e^{a t_{i}} \pi\left(x, q^{e}\left(t_{i}^{+}\right)\right)
$$

By Assumption A.2 any jump in $q^{e}(t)$ must be positive. Thus any jump in $\rho C-e^{a t_{i}} \pi\left(x, q^{e}\left(t_{i}^{-}\right)\right)$can only jump down if it jumps at all. Since $q^{e}\left(t_{1}^{+}\right) \leq q^{e}\left(t_{2}^{-}\right)$by A.2, we have a contradiction to eq. (A.1). To see this, consider the RHS of eq. (A.1) for $t_{1}$ :

$$
0 \geq \rho C-e^{a t_{1}} \pi\left(x, q^{e}\left(t_{1}^{+}\right)\right)>\rho C-e^{a t_{2}} \pi\left(x, q^{e}\left(t_{1}^{+}\right)\right)>\rho C-e^{a t_{2}} \pi\left(x, q^{e}\left(t_{2}^{-}\right)\right) \geq 0
$$

The second inequality between the 0 's follows because $t_{1}<t_{2}$ and the third inequality follows from monotonicity of $\pi(\cdot, \cdot)$ and $q^{e}\left(t_{1}^{+}\right) \leq q^{e}\left(t_{2}^{-}\right)$. We thus arrive at a contradiction. This argument addresses all four cases and ends the proof . 


\section{Proof of Lemma 2.}

Suppose by way of contradiction that $t<t_{1}$. Since $t_{1}$ is optimal,

$$
0 \leq g\left(x_{1}, t_{1}^{-}\right)=\rho C-\exp \left(a t_{1}\right) \pi\left(x_{1}, q^{e}\left(t_{1}^{-}\right)\right)<\rho C-\exp (a t) \pi\left(x, q^{e}\left(t^{+}\right)\right) \leq 0 .
$$

This chain of inequalities follows from optimality of $t_{1}$, the assumptions $t<t_{1}$ and $x<x_{1}$, and the implication of Assumption A.2 that $q^{e}\left(t_{1}^{-}\right) \geq q^{e}\left(t^{+}\right)$. As eq. (A.3) is internally contradictory, it must be the case that $t \geq t_{1}$.

\section{Proof of Theorem 1.}

We first prove that the candidate REE above is an REE. It will help to follow the argument if one considers the graph of $S(x)$ with $x$ on the horizontal axis and $S$ on the vertical axis.

Let $x_{i}(t), i=1,2,3$ denote the smallest, middle and largest solutions of the equation, $t=S(x)$; when the solution is unique, these solutions simply coincide. Note that for $t_{*}=S\left(x_{\min }\right), t^{*}=S\left(x_{\max }\right)$ there are two distinct values of $x, x_{*}$ and $x^{*}$ such that $t_{*}=S\left(x^{*}\right), t^{*}=S\left(x_{*}\right) . \quad$ Note that $x_{*}<x_{\min }<x_{\max }<x^{*} . \quad$ We will use the notation $x_{i}(t)=S_{i}^{-1}(t)$ when it is useful. For $t<t_{*}$, the large $x$ solution, $x_{3}(t)$ is the only solution of the equation $t=S(x)$, so intuitively it must be an REE, if an REE exists at all. For $t>t^{*}$ the small $x$ solution $x_{1}(t)$, is the only solution of the equation $t=S(x)$ so intuitively it must be an REE, if an REE exists at all. The first step in the proof of the theorem is to formally demonstrate that the large $x$ solution is part of an REE. The same type argument will also imply that the small $x$ solution is part of an REE. In other words, for each $x$, we 
need to prove that the optimal $t^{*}$ is the part of $S(x)$ described in the theorem statement. Since we showed in Lemma 1 that, for each fixed $x$, there can be at most one solution to the necessary conditions for a local maximum of $J(t, x, q)$ all we need to do is show that the relevant parts of $S(x)$ satisfy these necessary conditions for each $x$.

Consider first $x>x_{\max }$. By the implicit function theorem, the implicit function $S(z)-\tau=0$ has a well defined solution $z(\tau)=S_{3}^{-1}(\tau)$ in an open neighborhood of the point $(t, x)$ (given that $\left.S^{\prime}(x)<0\right)$. We claim that

$$
J^{\prime}(\tau, x)=e^{-\rho \tau}\left(\rho C-e^{a \tau} \pi\left(x, 1-F_{X}\left(S_{3}^{-1}(\tau)\right)\right)\right.
$$

equals 0 at $\tau=t=S(x)$ and is decreasing for all $\tau$ in an open neighborhood of $t$, i.e. $t$ is a local maximum of $J(\tau, x)$. Substituting $\tau=S(x)$ into $J(\tau, x)$ and using the definition of $S(x)$ and the identity $S_{3}^{-1}(S(x))=x$ it is evident that $J^{\prime}(\tau, x)=0$ at $\tau=t=S(x)$. Since $\pi(x . q)$ and $F_{X}(x)$ are, by assumption, continuously differentiable, and since by the implicit function theorem, $S_{3}^{-1}(z)$ is differentiable at $z=x$, it must be the case that $J(\tau, x, q)$ is differentiable at $\tau=t$. Since $S_{3}^{-1}(z)$ is strictly decreasing, we find by direct computation that $J^{\prime \prime}(\tau, x)<0$ at $\tau=t$ by using $J^{\prime}(\tau, x)=0$ at $\tau=t$. Hence the best reply property for an REE is satisfied for $x>x_{\max }$. The argument extends to $x=x_{\max }$ by taking right hand limits. Define $x_{*}<x_{\min }<x_{\max }$ to be the smaller value of the two values of $x$ that satisfy the equation $S\left(x_{\max }\right)=S\left(x_{*}\right)$. The same argument as above shows that the best reply property is satisfied for $x<X_{*}$.

We next verify that the optimal $t$ for $x \in\left[x_{*}, x_{\max }\right]$ is $t^{*}=S\left(x_{*}\right)=S\left(x_{\max }\right)$. We do $x_{\max }$ first. If one inserts $x=x_{\max }$, into $J^{\prime}$ evaluated at $t^{*}$, one sees immediately that 
$J^{\prime}\left(t^{*}, x_{\max }\right)=0$. If one replaces $t^{*}$ by a slightly smaller value of $t, J^{\prime}$ will be negative at $x=x_{\max }$ (on the branch $x_{3}(t)=S_{3}^{-1}(t)$ ).

We next consider $x_{*}$. This requires us to show that the derivative $J^{\prime}\left(t^{*}, x\right)$

$$
J^{\prime}\left(t^{*}, x\right)=e^{-\rho t^{*}}\left(\rho C-e^{-a t^{*}} \pi\left(x, q^{*}\left(t^{*}\right)\right)\right)
$$

Is positive (negative) for $t$ slightly less (greater) than $t^{*}$ for $t^{*}=\frac{1}{a} \ln \frac{\rho C}{\pi\left(x_{\max }, 1-F\left(x_{\max }\right)\right)}$. Now put $x=X_{*}$, but evaluate $J^{\prime}\left(t^{*}, x\right)$ in an interval around $t^{*}$, i.e. compute right limits and left limits at $t^{*}$. (Note that for the left limit we will be on the branch $q^{*}(s)=1-F_{X}\left(S_{3}^{-1}(s)\right), s<t^{*}$ and for the right limit we will be on the branch $\left.q^{*}(s)=1-F_{X}\left(S_{1}^{-1}(s)\right), s>t^{*}\right)$. The left limit as $\mathrm{t}$ approaches $t^{*}$ from below of $J^{\prime}\left(t^{*}, x, q^{*}\right)$ is easily seen to be given by

$$
J^{\prime}\left(t^{*-}, x_{*}\right)=e^{-\rho t^{*}} \rho C\left(1-\frac{\pi\left(x_{*}, 1-F_{X}\left(x_{\max }\right)\right)}{\pi\left(x_{\max }, 1-F_{X}\left(x_{\max }\right)\right)}\right)>0
$$

This same inequality holds for all $x \in\left[x_{*}, x_{\max }\right)$ since $\pi$ is assumed to be strictly increasing in $x$. Notice that $J^{\prime}\left(t^{*-}, x\right)$ above is the marginal gain to waiting an extra day to adopt before the jump in $q^{*}$.

Next, compute the right hand limit of $J^{\prime}(t, x)$ as $t$ approaches $t^{*}$ from above. The atom $F_{X}\left(x_{\max }\right)-F_{X}\left(x_{*}\right)$ has now adopted. Compute $J^{\prime}\left(t^{*}, x\right)$ at $t^{*}$ to obtain

$$
J^{\prime}\left(t^{*}, x_{*}\right)=e^{-\rho t^{*}} \rho C\left(1-\frac{\pi\left(x_{*}, 1-F_{X}\left(x_{*}\right)\right)}{\pi\left(x_{\max }, 1-F_{X}\left(x_{\max }\right)\right)}\right)=0
$$


because, by the definition of $x_{*}, \quad S\left(x_{*}\right)=S\left(x_{\max }\right)$ implies that $\pi\left(x_{*}, 1-F_{X}\left(x_{*}\right)\right)=\pi\left(x_{\max }, 1-F_{X}\left(x_{\max }\right)\right)$ and $S(x)=\frac{1}{a} \ln \frac{\rho C}{\pi\left(x, 1-F_{X}(x)\right)}$. We will be finished once we show that $J^{\prime}\left(t, x, q^{*}\right)$ is negative for $t$ slightly bigger than $t^{*}$. We have

$$
J^{\prime}\left(t^{*}+\varepsilon, x\right)=e^{-\rho\left(t^{*}+\varepsilon\right)} \rho C\left(1-\frac{\pi\left(x, q^{*}\left(t^{*}+\varepsilon\right)\right)}{\pi\left(x_{\max }, 1-F_{X}\left(x_{\max }\right)\right)}\right) .
$$

Define $h(\varepsilon)=1-\frac{\pi\left(x, q^{*}\left(t^{*}+\varepsilon\right)\right)}{\pi\left(x_{\max }, 1-F_{X}\left(x_{\max }\right)\right)}$. It is clear from (24) that the sign of $J^{\prime}\left(t^{*}+\varepsilon, x\right)$ must be the same as $h(\varepsilon)$. Since $h(\varepsilon)$ is continuously differentiable at $\varepsilon=0$, it has a Taylor expansion, $h(\varepsilon)=h(0)+h^{\prime}(0) \varepsilon+o(\varepsilon)$. We first show that $h(0)<0$ Recall that $\lim _{\varepsilon \rightarrow 0} q^{*}\left(t^{*}+\varepsilon\right)=1-F_{X}\left(x_{*}\right)$ because under $q^{*}$ the atom $F_{X}\left(x_{\max }\right)-F_{X}\left(x_{*}\right)$ is anticipated to have already adopted for dates $t$ slightly greater than $t^{*}$. From this, we obtain

$$
h(0)=1-\frac{\pi\left(x, 1-F_{X}\left(x_{*}\right)\right)}{\pi\left(x_{\max }, 1-F_{X}\left(x_{\max }\right)\right)}<0 .
$$

We next show that $h^{\prime}(0)<0$ This derivative equals

$$
h^{\prime}(0)=-a+\frac{\pi_{q}\left(x_{*}, 1-F_{X}\left(x_{*}\right)\right) f_{X}\left(x_{*}\right)}{S^{\prime}\left(x_{*}\right) \pi\left(x_{*}, 1-F_{X}\left(x_{*}\right)\right)}<0
$$

which is negative because $S^{\prime}\left(x_{*}\right)<0$. This completes the proof for $x=X_{*}$. 
The proof for each $x \in\left(x_{*}, x_{\max }\right)$ proceeds as follows. We have already shown that the left hand limit of $J^{\prime}\left(t^{*}, x\right)$ is positive for each such $x$. We must now show that the right hand limit of $J^{\prime}\left(t^{*}, x\right)$ is negative for each such $x$. We have

$$
J^{\prime}\left(t^{*}+\varepsilon, x\right)=e^{-\rho\left(t^{*}+\varepsilon\right)} \rho C\left(1-\frac{\pi\left(x, q^{*}\left(t^{*}+\varepsilon\right)\right)}{\pi\left(x_{\max }, 1-F_{X}\left(x_{\max }\right)\right)}\right)
$$

Define $h(\varepsilon)=1-\frac{\pi\left(x, q^{*}\left(t^{*}+\varepsilon\right)\right)}{\pi\left(x_{\max }, 1-F_{X}\left(x_{\max }\right)\right)}$. We show that $h(0)<0 . \quad$ Recall that $\lim _{\varepsilon \rightarrow>0} q^{*}\left(t^{*}+\varepsilon\right)=1-F_{X}\left(x_{*}\right)$ because under $q^{*}$ the atom $F_{X}\left(x_{\max }\right)-F_{X}\left(x_{*}\right)$ is anticipated to have already adopted for dates $t$ slightly greater than $t^{*}$. From this, we obtain

$$
h(0)=1-\frac{\pi\left(x, 1-F_{X}\left(x_{*}\right)\right)}{\pi\left(x_{\max }, 1-F_{X}\left(x_{\max }\right)\right)}<0
$$

because $\pi\left(x_{\max }, 1-F_{X}\left(x_{\max }\right)\right)<\pi\left(x, 1-F_{X}(x)\right)<\pi\left(x, 1-F_{X}\left(x_{*}\right)\right)$ for $x \in\left(x_{*}, x_{\max }\right)$. This completes the proof .

\section{Proof of Theorem 2.}

If there are no social interactions, $\pi(x, q)=\pi(x)$. Hence the equation $t=S(x)=a^{-1} \ln \left(\frac{\rho C}{\pi(x)}\right)$ has a unique solution for each $t$. Denote $q_{i}(t)=1-F_{X, i}\left(S^{-1}(t)\right)$. Therefore, $q_{1}(t)=1-F_{X, 1}\left(S^{-1}(t)\right) \leq q_{2}(t)=1-F_{X, 2}\left(S^{-1}(t)\right)$, 
by stochastic dominance .

\section{Proof of Theorem 3.}

The proof of the first part is obtained from a straightforward chain of inequalities that exploits the assumption that $\pi=\pi\left(x_{1}, x_{2}\right)$ is independent of $q$ and is strictly increasing in both $x^{\prime} \mathrm{s}$ as well as Assumption A.5, Formally,

$$
\begin{gathered}
q\left(t \mid x_{1}\right)=1-F_{X_{2} \mid X_{1}}\left(S^{-1}\left(t \mid x_{1}\right) \mid x_{1}\right) \leq \\
1-F_{X_{2} \mid X_{1}}\left(S^{-1}\left(t \mid x_{1}\right) \mid x_{1}^{\prime}\right)<1-F_{X_{2} \mid X_{1}}\left(S^{-1}\left(t \mid x_{1}^{\prime}\right) \mid x_{1}^{\prime}\right)=q\left(t \mid x_{1}^{\prime}\right)
\end{gathered} .
$$

The first inequality follows from eq. (22) and the second follows from $S^{-1}\left(t \mid x_{1}\right)>S^{-1}\left(t \mid x_{1}^{\prime}\right)$, which follows from $S\left(x_{2} \mid x_{1}\right)>S\left(x_{2} \mid x_{1}^{\prime}\right)$, an inequality which follows directly from the assumption that $\pi\left(x_{1}, x_{2}\right)$ is strictly increasing in the vector $\left(x_{1}, x_{2}\right)$. Given the proof of the first part, the proof of the second part is immediate. This ends the proof . 


\section{Appendix 2 : Closed Form Example For Theorem 1.}

Here we construct a parametric example of an environment of the type assumed in Theorem 1. First, note that $S(x)=a^{-1} \ln \frac{\rho C}{\pi(x, q(x))}$ falls, then rises, then falls again if and only if $\pi(x, q(x))$, where $q(x)=1-F_{X}(x)$ moves in the opposite way, i.e. rises, then falls, then rises. We therefore construct a $\pi(x, q(x))$ and $q(x)=1-F(x)$ that satisfies these last properties. We will choose $A_{1}>0$ and construct two functions with the first function designed so that it is concave, continuously differentiable, and increases then decreases on $\left(0, A_{1}\right)$ and the second function defined on $\left[A_{1}, \infty\right)$ such that they join together to form a continuous function. The parameters of these functions will be designed so they generate an example of Theorem 1. This exercise will prove that Theorem 1 applies to a nontrivial set of examples.

We define the first function by

$$
\pi_{1}(x, q(x))=x^{\alpha}(K+B(1-F(x)))^{\beta}
$$

where $\alpha>0, \beta>0, \alpha+\beta=1, K>0, B>0$ and $F_{X}(x)$ are chosen so that this function is continuously differentiable, concave, increases, takes a maximum, call it $x_{\min }$ (because it is a local minimum of $S(x)$, on $\left(0, A_{1}\right)$, for appropriately chosen $A_{1}>0$, and decreases to the smaller value $\pi_{1}\left(A_{1}, q\left(A_{1}\right)\right)<\pi_{1}\left(x_{\min }, q\left(x_{\text {min }}\right)\right)$. Clearly $\pi(0, q(0))=0$. As we will see below, this function will be concave, will increase to a maximum, then take a smaller value at $\pi\left(A_{1}, 1-F_{X}\left(A_{1}\right)\right)$ for appropriately chosen linear $F_{X}(x)$ on $\left(0, A_{1}\right)$.

Our second function will be defined by

$$
\pi_{2}\left(x, 1-F_{X}(x)\right)=\pi_{1}\left(A_{1}, 1-F_{X}\left(A_{1}\right)\right)+L_{1}\left(x-A_{1}\right)+L_{2}\left(F_{X}\left(A_{1}\right)-F_{X}(x)\right)
$$


where $A_{2}>A_{1}, F_{X}(x)$ is chosen to be linear, and the other parameters are chosen so that $\pi_{2}\left(x, 1-F_{X}(x)\right)$ increases on $\left[A_{1}, \infty\right)$ and $\pi_{2}\left(A_{2}, 1-F_{X}\left(A_{2}\right)\right)>\pi_{1}\left(x_{\min }, 1-F_{X}\left(x_{\min }\right)\right)$. Note that the rightmost term comes from the term $L_{2}\left(q(x)-q\left(A_{1}\right)\right)$ and that the two functions take the same value at $x=A_{1}$. Therefore the join is continuous at $x=A_{1}$. We also choose $F_{X}(x)$ so that it has positive support on $\left[0, x_{S}\right), x_{S} \geq A_{2}$

Return to the specification of the first function. Compute

$$
\begin{gathered}
\frac{d \pi_{1}(x, q(x))}{d x}= \\
\alpha x^{\alpha-1}\left(K+B\left(1-F_{X}(x)\right)^{\beta}-\beta x^{\alpha}\left(K+B\left(1-F_{X}(x)\right)\right)^{\beta-1} B f_{X}(x)=\right. \\
\pi_{1}(x, q(x))\left(\frac{a}{x}-\frac{\beta B f_{X}(x)}{K+B\left(1-F_{X}(x)\right)}\right)
\end{gathered}
$$

It is evident that this derivative is positive for small $x$, is 0 for some $x_{\min }>0$, and is negative for larger $x$ 's iff $\left(\frac{a}{x}-\frac{\beta B f_{X}(x)}{K+B\left(1-F_{X}(x)\right)}\right)$ has the same properties. We therefore choose $F_{X}(x)$ to satisfy,

$$
\begin{gathered}
F_{X}(x)=\frac{x}{a_{1}}, x \in\left[0, A_{1}\right), \\
=\frac{A_{1}}{a_{1}}+\frac{x-A_{1}}{a_{2}} x \in\left[A_{1}, A_{2}\right), \\
=1, x \geq A_{2}
\end{gathered}
$$

where we are free to choose all these parameters to get what we want and satisfy all the explicit and implicit constraints needed to get an example with just one jump. It is easy to 
check that $\pi_{1}$ rises for small $x^{\prime}$ s, takes a first zero at $x_{\min }=\frac{\alpha}{\alpha+\beta} \frac{a_{1}(K+B)}{B}$, and is concave on $\left(0, A_{1}\right)$ so our first constraint on our parameter set is that $x_{\min }<A_{1}$.

Without loss of generality recall that we can assume that $\alpha+\beta=1$ by taking a monotonic transformation. Therefore we know that $\pi_{1}$ is concave on $\left(0, A_{1}\right)$ so we know that we have identified a maximum on $\left(0, A_{1}\right)$. We now wish to construct the "rest" of $F_{X}(x)$ and the function $\pi_{2}$ to produce a local minimum of $\pi$ at $A_{1}$ and such that for some $A_{2}>A_{1}$, we have $\pi_{2}\left(A_{2}, 1-F_{X}\left(A_{2}\right)\right)>\pi_{1}\left(x_{\min }, 1-F_{X}\left(x_{\min }\right)\right)$ before the end of the support of $F_{X}(x)$ is reached. To do this, we identify parameters so that there is a local minimum of $\pi$ at $x=A_{1}$. We already know that the left hand derivative of $\pi=\pi_{1}$ at $x=A_{1}$ is negative. We must specify the parameters of $\pi_{2}$ so that the right hand derivative of $\pi=\pi_{2}$ is positive at $x=A_{1}$. This condition is satisfied if $L_{1}-\frac{L_{2}}{a_{2}}>0$.

Finally we require $\frac{A_{1}}{a_{1}}<1$ and $\frac{A_{1}}{a_{1}}+\frac{A_{2}-A_{1}}{a_{2}} \leq 1$. Since $S(x)=\frac{1}{a} \ln \left(\frac{\rho C}{\pi(x, q(x))}\right)$,

we may choose $\rho C$ large enough so that it is above $\pi$ evaluated at $x_{\min }$ so that $S\left(x_{\min }\right)>0$. We still have quite a bit of freedom to construct the rest of the example to illustrate Theorem 1. Note that we have a nondifferentiability of $S(x)$ at $x=A_{1}$ whereas Theorem 1 assumes continuous differentiability of $S(x)$. But the proof uses left and right limits so differentiability is not really needed. In any event one can always locally smooth the construction at $x=A_{1}$.

We still need to choose the parameters so that the local maximum value of $\pi$ is smaller than $\pi\left(A_{2}, 1-F_{X}\left(A_{2}\right)\right)$ so that we have a positive atom for our jump. This is satisfied provided that 


$$
\begin{gathered}
\pi_{2}\left(A_{2}, 1-F_{X}\left(A_{2}\right)\right)= \\
\pi_{1}\left(A_{1}, 1-F_{X}\left(A_{1}\right)\right)+L_{1}\left(A_{2}-A_{1}\right)+L_{2}\left(F_{X}\left(A_{1}\right)-F_{X}\left(A_{2}\right)\right)>\pi_{1}\left(x_{\text {min }}, 1-F_{X}\left(x_{\text {min }}\right)\right)
\end{gathered}
$$

For simplicity, assume $0<a_{1}=a_{2}$. The restrictions needed on the various parameters may be summarized as

$$
\frac{A_{1}}{a_{1}}<1, \frac{\alpha a_{1}(K+B)}{B}<A_{1}, \frac{A_{2}}{a_{1}} \leq 1, L_{1}-\frac{L_{2}}{a_{1}}>0
$$

and

$$
\begin{gathered}
L_{1}\left(A_{2}-A_{1}\right)+\frac{L_{2}\left(A_{1}-A_{2}\right)}{a_{1}}= \\
\left(L_{1}-\frac{L_{2}}{a_{1}}\right)\left(A_{2}-A_{1}\right)>\pi_{1}\left(x_{\min }, 1-\frac{x_{\min }}{a_{1}}\right)-\pi_{1}\left(A_{1}, \frac{1-A_{1}}{a_{1}}\right)
\end{gathered}
$$

The approach we have outlined provides a method for the construction of a fairly large class of examples. The construction illustrates that it is straightforward to construct a class of examples that satisfy the conditions of Theorem 1 so that there is an atom jump. For example if one assumes that $K=0$ and $B=1$, it is clear that one can set $L_{1}$ large enough and $L_{2}$ small enough to satisfy all the above constraints. Define

$$
\begin{gathered}
S(x) \\
=\frac{1}{a} \ln \left(\rho C / \pi_{1}\left(x, 1-\frac{x}{a_{1}}\right)\right), \text { if } x<A_{1} \\
=\frac{1}{a} \ln \left(\rho C / \pi_{2}\left(x, 1-\frac{x}{a_{1}}\right)\right) \text { if } x \geq A_{1}
\end{gathered}
$$


Thus, $t^{*}=S\left(A_{1}\right)$, by continuity there is $x_{*}<x_{\min }<A_{1}, t^{*}=S\left(x_{*}\right)=S\left(A_{1}\right)$. The size of the jump atom is $F_{X}\left(A_{1}\right)-F_{X}\left(x_{*}\right)=\frac{A_{1}-x_{*}}{a_{1}}$.

The lack of differentiability of the example at $x=A_{1}$ is not a problem since we did not actually use differentiability in the proof of Theorem 1. Furthermore, the example can be smoothed in an arbitrarily small neighborhood of $x=A_{1}$ by choosing smooth functions for each side of $x=A_{1}$. 


\section{Bibliography}

Adsera, A. and D. Ray, (1998), "History and coordination failure," Journal of Economic Growth, 3, 267-276.

Ait-Sahalia, Y., (2004), "Disentangling diffusion from jumps," Journal of Financial Economics, 74, 487-528.

Ait-Sahalia, Y. and J. Jacod, (2008), “Testing for jumps in a discretely observed process," Annals of Statistics, forthcoming.

Bandiera, O., Rasul, I., (2006), "Social networks and technology adoption in northern Mozambique,” Economic Journal, 116, 869-(092.

Barndoff-Neilson, O. and N. Shephard, (2006), "Econometrics of testing for jumps in financial economics using bipolar variation,” Journal of Financial Econometrics, 4, 1-30.

Brock, W., (1999), "Scaling in economics: A reader's guide," Industrial and Corporate Change, 8, 409-446.

Brock, W., (2001), "Complexity-based methods in cycles and growth: Any potential valueadded?” in Punzo, L., pp. 301-338.

Brock, W. and S. Durlauf, (2001a), "Discrete choice with social interactions," Review of Economic Studies, 68, 235-260.

Brock, W. and S. Durlauf, (2001b), "Interactions-based models," in: J. Heckman and E. Leamer, eds., Handbook of Econometrics, Volume 5, North-Holland: Amsterdam, 32973380 .

Brock, W. and S. Durlauf, (2006), "Multinomial choice with social interactions," in The Economy as an Evolving Complex System III, L. Blume and S. Durlauf, eds., New York: Oxford University Press.

Brock, W. and S. Durlauf, (2007), "Identification of binary choice models with social interactions," Journal of Econometrics, 140, 52-75.

Cabral, L., (1990), "On the adoption of innovations with 'network' externalities," Mathematical Social Sciences, 19, 299-308.

Conley, T. and C. Udry, (2005), "Learning about a new technology: Pineapple in Ghana," Working paper, University of Chicago and Yale University.

Daley, D. and J. Gani, (2001), Epidemic Modelling, New York: Cambridge University Press. 
David, P., (2005), "Zvi Griliches on diffusion, lags and productivity growth...connecting the dots,” mimeo, Stanford University.

De Palma, A., (2007), "Inference in a synchronization game with social interactions," mimeo, University of Pennsylvania.

DiNardo, J. and J. Winfree, (2007), “The law of genius and home runs refuted,” mimeo, University of Michigan.

Feller, W., (1940), "On the logistic law of growth and its empirical verification in biology," Acta Biotheoretica, 5, 51-66.

Graham, B., (2008), "Identifying social interactions through conditional variance restrictions," 76, 3, 643-660.

Griliches, Z., (1957), "Hybrid corn: An exploration of the economics of technical change," Econometrica, 25, 501-522.

Griliches, Z., (1958), "Research costs and social returns: Hybrid corn and related interactions," Journal of Political Economy, 66, 419-431.

Havens, E. and E. Rogers, (1961), "Profitability and the interaction effect," Rural Sociology, 26, 409-414.

Lee, L.-F., (2007), "Identification and estimation of econometric models with group interactions, contextual factors and fixed effects," Journal of Econometrics, 140, 333-374.

Manski, C., (1993), "Identification of endogenous social effects: The reflection problem," Review of Economic Studies, 60, 531-42.

Manski, C., (1997), "Monotone treatment response,” Econometrica, Society, 65, 6, 1311 1334.

Manski, C., (2004), "Social learning from private information: The dynamics of the selection problem,” Review of Economic Studies, 71, 443-458.

Manski, C., (2006), "Social learning and the adoption of innovations," in Blume L., Durlauf, S., eds., The Economy as an Evolving Complex System, III, Oxford University Press: Oxford.

Manski, C. and J. Pepper, (2000), "Monotone instrumental variables with an application to the returns to schooling," Econometrica, 68, 997-1010.

Manuelli, R. and A. Seshadri, (2008), "Frictionless technology diffusion: the case of tractors," mimeo, Univeresity of Wisconsin. 
Munshi, K., (2004), "Social learning in a heterogeneous population: technology diffusion in the Indian Green Revolution,” Journal of Development Economics, 73, 185-213.

Punzo, L., ed., (2001), Cycles, Growth, and Structural Change: Theories and Empirical Evidence, Routledge: New York.

Reinganum, J., (1981a), "On the diffusion of new technology: A game theoretic approach," Review of Economic Studies, 98. 395-405.

Reinganum, J., (1981b), "Market structure and the diffusion of new technology," Bell Journal of Economics, 12, 2, 618-624.

Schelling, T., (1997), "Social mechanisms and social dynamics," in Social Mechanisms, P. Hedstrom and R. Swedberg, eds., New York: Cambridge University Press.

Sirakaya, S. (2006), "Recidivism and social interactions," Journal of the American Statistical Association, 101, 475, 863-877.

Skinner, J. and D. Staiger, D., (2005), "Technology adoption from hybrid corn to beta blockers," National Bureau of Economic Research Working Paper no. 11251.

Sutch, R., (2008), "Henry Agard Wallace, the Iowa corn yield tests, and the adoption of hybrid corn,” National Bureau of Economic Research Working Paper no. 14141.

Varian, H., (1992), Microeconomic Theory, New York: Norton.

Young, H. P., (2007), "Innovation diffusion in heterogeneous populations," mimeo, University of Oxford. 